\title{
THREE-DIMENSIONAL ANALYSIS OF KINEMATIC AND KINETIC COORDINATION OF THE LOWER LIMB JOINTS DURING STAIR ASCENT AND DESCENT
}

\author{
Hsiu-Chen Lin', Tung-Wu Lu', Horng-Chaung Hsu² \\ 'Institute of Biomedical Engineering, National Taiwan University, Taipei, Taiwan. \\ ${ }^{2}$ Department of Orthopedics, China Medical University Hospital, Taichung, Taiwan.
}

\begin{abstract}
Being a common daily activity, stair locomotion places much higher loads on the lower limb than level walking does so a better understanding of the biomechanics of this activity is important for evaluation and treatment for patients with lower limb problems. The purpose of the present study was to investigate the three-dimensional dynamics and coordination of the joints of the lower limb during the stance phase of stair ascent and descent. Ten normal young adult subjects were recruited to ascend and descend stairs in a gait laboratory where the three-dimensional kinematic and kinetic data as well as muscle electromyography (EMG) were collected. The sagittal ranges of motion during stance phase of stair ascent were from $1.85^{\circ}$ extension to $53.5^{\circ}$ flexion for the hip, $13.1^{\circ}$ to $60.1^{\circ}$ flexion for the knee and $13.8^{\circ}$ dorsiflexion to $14.0^{\circ}$ plantarflexion for the ankle. Corresponding data for stair descent were 4.78( to 13.16( flexion for the hip. 8.3. to $77.6^{\circ}$ flexion for the knee and $18.3^{\circ}$ dorsiflexion to $27.4^{\circ}$ plantarflexion for the ankle. Maximum extensor moments of $8.5 \%$ and $15.6 \%(\mathrm{Nm} / \mathrm{BW} / \mathrm{LL})$ were required at the hip and knee respectively during loading response while $19.4 \%(\mathrm{Nm} / \mathrm{BW} / \mathrm{LL})$ at the ankle shortly before toe-off. During stair descent. maximum extensor moments of about $4.4 \%$ were required at the hip during loading response and before toe-off while $13.3 \%$ and $15.2 \%(\mathrm{Nm} / \mathrm{BW} / L \mathrm{~L})$ at the knee and ankle respectively before toe-off. The joint angles. moments, and powers in the frontal and transverse planes were relatively small, except for hip abduction. The hip abductor moments and powers were significantly bigger than those of the knee and ankle in both stair activities. Joint powers and the corresponding muscle activation patterns in stair ascent were significantly different from those in descent, with concentric powers generated mostly during stair ascent and with eccentric powers stair descent. The differences of the movements of the lower limb during stair ascent and descent were due to different safety requirements and kinematic and kinetic constraints from the stairs. The complete description of the biomechanics of the lower extremity while performing stair locomotion will be helpful for the planning and evaluation of treatment programs for patients with lower limb problems.
\end{abstract}

Biomed Eng Appl Basis Comm, 2004(April); 16: 101-108.

Keywords: gait analysis, lower limb, stair ascent, stair descent, EMG

Received: Jan 7, 2004; Accepted: Mar 4, 2004

Correspondence: Tung-Wu Lu, Professor

Institutc of Biomedical Engineering, National Tajwan University, Tajpci 100, Taiwan

E-mail: twiu@ntu.edu.tw

\section{INTRODUCTION}

Stair locomotion is common functional activity of daily living. It is also used in rehabilitation as a multijoint close kinetic chain (CKC) exercise. Stair locomotion placed much higher loads on the lower 
limb than level walking does, with larger ranges of motion and muscle efforts in the joints of the lower limbs. Therefore, a better understanding of the biomechanics of the lower extremity while performing this activity is important for planning and evaluation of treatment programs for patients with lower limb problems.

Computer-aided three-dimensional motion analysis has been used extensively in investigating movement patterns during various functional activities in different populations of subjects. Early studies on stair activities in normal subjects using motion analysis techniques were mostly limited to two-dimensional (2D) movement patterns [1-3]. Andriacchi et al. [1] described the sagittal kinematics of the lower limb and the corresponding phasic muscle activities in one subject during going up and down stairs. Threedimensional net external moments were also given. McFadyen and Winter [2] reported joint angles, moments, supporting moments and joint powers in the sagittal plane as well as electromyographic (EMG) data from three normal male subjects during stair locomotion. Livingston et al. [3] investigated the influence of stair dimension on temporal variables and joint kinematics with one high-speed camera and suggested that individuals accommodated these environmental changes mainly by knee flexion. Zachazewski et al. [4] described detailed temporal phases of stair locomotion identified with ground reaction forces and trajectories of the body centre of mass (COM). This study considered the overall body movement during stair locomotion but motion of individual joints of the lower limb were not given. Biplanar (sagittal and coronal) analyses of stair locomotion existed but have been limited to the knee joint because of interests on patients with knee pain, ACL injuries or osteoarthritis [5-6]. Recently, Costigan et al. [7] used a subject-specific knee model to calculate 3D knee kinetics during stair ascent and investigated its interaction with the hip but the movement at the ankle joint and the foot were ignored. Kaufman et al. [8] studied the 3D kinematics and kinetics of the lower limb during stair ascent and descent in patients with knee OA but unfortunately only data for the knee joint in normal subjects were reported.

Stair locomotion is predominately a multi-joint movement so a complete knowledge on the coordination and mechanical interactions among the joints is essential. Although previous studies helped in establishing a general picture of the movement patterns and strategies utilized during stair locomotion in single planes, more insight into the biomechanics of this activity for future clinical applications requires a detailed three-dimensional (3D) analysis of the individual joints of the lower extremity. The purpose of the present study was thus to provide a complete description of the three-dimensional dynamics and coordination of the joints and muscles of the lower limb during the stance phase of stair locomotion.

\section{MATERIALS AND METHODS}

Ten normal young adults (five males and five females; age: $21.9 \pm 1.6$ years; height: $167.9 \pm 8.3 \mathrm{~cm}$; weight: $61.5 \pm 9.8 \mathrm{~kg}$ ) were recruited in this study with informed consents. They were free of low back problem or other knee pathology that would affect their functional activities.

A marker system with 26 markers was developed for the description of the motion of the lower limb segments. Markers were attached to specific bony landmarks on each limb, including anterior superior iliac spines (ASIS), posterior superior iliac spines (PSIS), greater trochanter, mid-thigh, medial and lateral femoral epicondyles, tibial tuberosity, head of fibula, medial and lateral malleoli, calcaneus, navicular tuberosity and the base of the fifth metatarsal. Threedimensional coordinates of the markers during a static standing trial were collected for each subject to define the body-embedded coordinate systems for the segments. The markers on the medial femoral epicondyle and medial malleolus were removed during dynamic trials to prevent from interfering the movements.

Each subject was asked to climb a custom-made three-step stair (height: $18 \mathrm{~cm}$; depth: $46 \mathrm{~cm}$ ) first ascending and then descending with both legs tested (Fig. 1). A seven-camera motion analysis system (VICON 370, Oxford Metrics, U.K.) was used to measure the trajectories of the markers with a sampling rate of $60 \mathrm{~Hz}$. The ground reaction forces (GRF) were measured at a sampling rate of $240 \mathrm{~Hz}$ with two force platforms (AMTI, Mass., U.S.A.), which served as the second step of the three-step stair (Fig. 1). The starting position of the subject was adjusted by the examiner so that the tested foot could place naturally on the force platform during tested activities. A 10-channel electromyography (EMG) system (MA-300, Motion Lab Systems, U.S.A) with pre-amplified electrodes was used to record simultaneously activities of eight muscles in the right limb, namely gluteus maximus, rectus femoris, vastus medialis, vastus lateralis, medial hamstrings, biceps femoris, tibialis anterior and medial gastrocnemius. The EMG of the muscles during maximum voluntary isometric contractions (MVIC) was also collected for each subject before stair locomotion tests. Motion data from at least 3 trials for each test were collected.

The body-embedded coordinate system for each segment was defined using markers on that segment, 


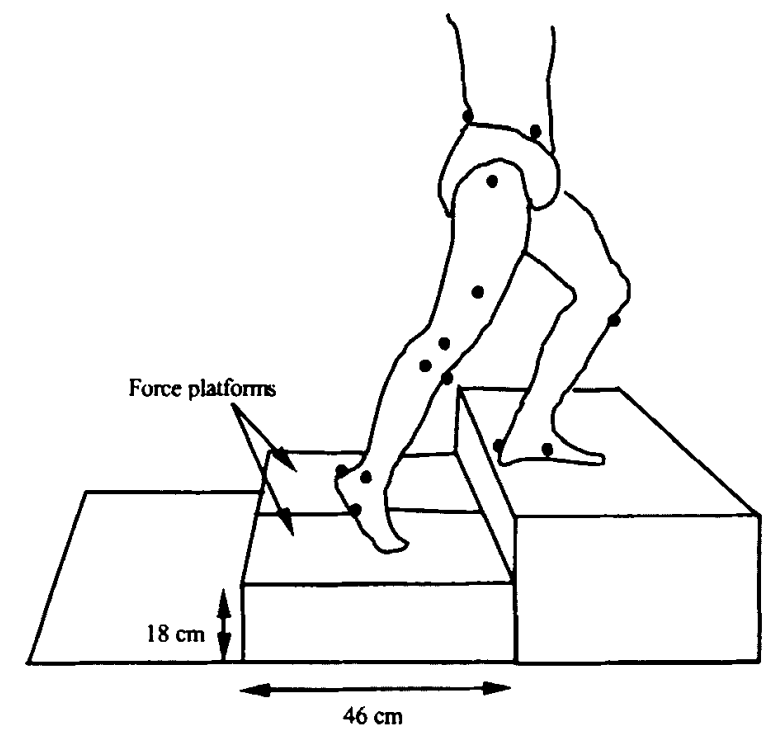

Fig. 1. Schematic diagram showing a subject ascending a three-step stair with the tested foot stepped on the second step fitted with two force platforms. Part of markers placed on the bony landmarks for the tracking of the limb segments is also shown.

with the positive $\mathrm{x}$ axis directed anteriorly, $\mathrm{y}$ axis superiorly and $z$ axis to the right. Rotational movements of the joints were described using Cardanic rotation sequences following recommendations by Grood and Suntay [9]. The corresponding clinical terms for the calculated Cardanic angles were assigned following the recommendations from the Standards and Terminology Committee of the International Society of Biomechanics [10]. Joint angles, moments and powers were calculated using inverse dynamics techniques with a 3-D lower limb model from the measured kinematic and kinetic data. Inertial properties of each segment were determined using Dempster's coefficients [11]. Joint moments were then normalized to the body weight and leg length and joint powers to body weight for each subject. Raw EMG was first filtered with a notch filter of $60 \mathrm{~Hz}$ and then a band-pass filter of $40 \mathrm{~Hz}$ to $240 \mathrm{~Hz}$ to eliminate noises and motion artefacts. The resulting EMG data were rectified and low-pass filtered with a 5th-order Butterworth filter with a cut-off frequency of $10 \mathrm{~Hz}$ to obtain the linear envelopes, which were then normalized with the MVIC to obtain the phasic activity of the muscles. Values of each variable from all the subjects were ensemble-averaged and the obtained mean and standard deviation were then used for subsequent description of the joint motions.

\section{RESULTS}

\subsection{Stair Ascent}

During the stance phase of stair ascent, the maximum mean flexion angles of the hip and knee occurred at initial contact, which were $53.5^{\circ}$ and $60.1^{\circ}$, respectively (Fig. 2a-b). Immediately after initial contact and during loading response $(0-25 \%$ cycle), the joints remained more or less at the same position, sustaining extensor moments as high as $8.5 \%$ $(\mathrm{Nm} / \mathrm{BW} / \mathrm{LL})$ at the hip, $15.6 \%(\mathrm{Nm} / \mathrm{BW} / \mathrm{LL})$ at the knee and $4 \%(\mathrm{Nm} / \mathrm{BW} / \mathrm{LL})$ at the ankle (Fig. 3a-c). The extensors of the hip and knee joints generated large concentric powers up to $10.7 \%$ and $24.9 \%$ (Watt/BW) while virtually none at the ankle, Fig. 4a-b. At about the same time, EMG evidence showed that gluteus maximus, rectus femoris, and the vasti were active and reached their peaks, Fig. 5a-d. After 25\% cycle, both the hip and knee joints extended gradually and reached their minima of about $18.8^{\circ}$ and $24.6^{\circ}$ of flexion, respectively, at the end of the first half of the stance phase (Fig. 2a-b). The ankle joint remained at about the same dorsiflexed position $\left(5.3-13.78^{\circ}\right)$, Fig. 2c. A relatively small ankle plantarflexor moment was needed at the ankle in the first half of the stance phase. The hamstrings and tibialis anterior were active during this period (Fig. 5e-g).

In the second half of the stance phase, the knee remained at about the same position, extending from $24.6^{\circ}$ to $10.3^{\circ}$ of flexion, Fig. $2 \mathrm{~b}$. The hip extended continuously from 18.8 ( of flexion to $2.2^{\circ}$ of extension, Fig. 2a. The ankle remained dorsiflexed until at $80 \%$ cycle where plantarflexion began and continued to a maximum of $14.0^{\circ}$ at the end of the stance phase (Fig. 2c). The hip joint continued to sustain relatively small extending moment during this period except for a burst of hip extensor moment appearing before toe-off (Fig. 3a). For the knee joint, flexor moments were required, Fig. 3b. An increasing plantarflex or moment with a peak of $19.35 \%$ $(\mathrm{Nm} / \mathrm{BW} / \mathrm{LL})$ at the ankle was required for pushing-off the body around toe-off (Figs. 2c, 3c). The corresponding peak angular power was $33.9 \%$ (Watt/BW). EMG data showed that the gastrocnemius was recruited to provide this propulsion power. The tibilalis anterior remained $20 \%$ activated for nearly all the stance phase.

In the frontal and transverse planes, the ranges of motion of the three joints were mostly within $10^{\circ}$. The hip joint kept adducted up to $4.5^{\circ}$ throughout the first half of the stance phase and then remained at neutral position until the propulsion of the body started at about $75 \%$ cycle when the hip joint began abduction and reached $4.2^{\circ}$ around toe-off (Fig. 2d). The knee joint was adducted to $1.3^{\circ}$ at initial contact and during 
the subsequent loading response and then remained in less than $2.6^{\circ}$ of abduction throughout the stance phase (Fig. 2e). Both the hip and knee joints remained externally rotated throughout the stance phase (Fig. $2 \mathrm{~g}$ h). The external rotation angles ranged from $0.5^{\circ}$ to $3.2^{\circ}$ for the hip and $2.0-11.5^{\circ}$ for the knee. The ankle was at neutral position at initial contact and then remained everted until about $80 \%$ cycle. During the last $20 \%$ cycle, a slight inversion $\left(4.5^{\circ}\right)$ combined with internal rotation $\left(6.3^{\circ}\right)$ were required at the foot for propulsion of the body (Fig. 2f). Even with relatively small ranges of motion in the frontal and transverse planes, significant moments were found at the joints. Abductor moments at the hip were required during the stance phase. These moments had a two-peak pattern with peaks of up to $8.8 \%(\mathrm{Nm} / \mathrm{BW} / \mathrm{LL})$ (Fig. $3 \mathrm{~d})$. The hip internal rotators exerted a moment of up to $3.9 \%$ $(\mathrm{Nm} / \mathrm{BW} / \mathrm{LL})$ at loading response, which were then decreased gradually for the rest of the first half of stance phase and reached to near zero at about $50 \%$ cycle. During the second half of the stance phase, the internal/external rotator moments were less significant, Fig. 3g. The knee abductor moment emerged after loading response, reaching a maximum of up to $3.3 \%$ $(\mathrm{Nm} / \mathrm{BW} / \mathrm{LL})$ at $75 \%$ cycle for propulsion, and then decreased to zero at toe-off (Fig. 3e). The external rotator moments at the ankle were around zero during the first $75 \%$ cycle and exerted a small peak of $3.8 \%$ (Nm/BW/LL) afterwards for body propulsion (Fig. 3i). Joint angular powers were all insignificant except for the hip abductors, which exerted concentric power as high as $2.5 \%$ (Watt/BW) after the loading response throughout the stance phase (Fig. 4d).

\subsection{Stair Descent}

During stair descent, the hip joint angles in the sagittal plane ranged between $4.8^{\circ}$ and $13.2^{\circ}$ throughout stance phase (Fig. 2a). The hip extensor moments, provided predominately by the gluteus maximus, were required during loading response $(0$ $20 \%)$ and late stance $(80 \%-100 \%)$ and generating corresponding concentric power $(0-10 \%)$ and eccentric power (90-100\%) (Fig. 3a).

The flexion angle of the knee joint was $8.3^{\circ}$ at initial contact and up to $26.4^{\circ}$ during loading response and remained at about $25^{\circ}$ during $20-50 \%$ cycle. In the second half of the stance phase, the knee joint flexed further and reached a maximum flexion of $76.6^{\circ}$ at toe-off, Fig. 2b. The extensor moments of the knee had two peaks, the first being $9.3 \%(\mathrm{Nm} / \mathrm{BW} / \mathrm{LL})$ and the second $13.3 \%(\mathrm{Nm} / \mathrm{BW} / \mathrm{LL})$, Fig. $3 \mathrm{~b}$. The curves for extensor activation and those for the corresponding joint moments and eccentric powers at the knee were found to be similar in shape. The co-contraction of rectus femoris and biceps femoris was found in the first half of the stance phase (Fig. 5b, $5 \mathrm{f})$. The quadriceps was active throughout the second half of the stance phase, with its maximum contraction occurred around $70 \%$ cycle (Fig. 5b-d).

The ankle joint was $18.3^{8}$ plantarflexed at initial contact and then rapidly flexed to around $10^{\circ}$ of dorsiflexion during the first $20 \%$ cycle. After $20 \%$ cycle, the ankle gradually dorsiflexed and reached a maximum of $21^{\circ}$ dorsiflexion at $85 \%$ cycle, followed by plantarflexion to toe-off with a final dorsiflexed position of $6.2^{\circ}$ (Fig. 2c). The sagittal ankle joint moment curve had two peaks, amplitudes of which were $10.8 \%(\mathrm{Nm} / \mathrm{BW} / \mathrm{LL})$ for the first and $15.2 \%$ (Nm/BW/LL) for the second (Fig. 3c). From the EMG data, co-contraction of tibialis anterior and gastrocnemius was found during the first $20 \%$ cycle. The activation level of the gastrocnemius decreased after $20 \%$ cycle while tibilis anterior remained activated at about the same level throughout the cycle, Fig. 5g-h. The gastrocnemius muscle provided an eccentric plantarflexor power of up to $31.7 \%$ (Watt/BW) during the first $20 \%$ cycle, corresponding to the building up of the first peak of the joint moment. The tibilis anterior provided an eccentric dorsiflexor power of up to $20.3 \%$ (Watt/BW) around toe-off, corresponding to the building up of the second peak of the joint moment (Fig. 4c).

The ranges of motion of the three joints in the frontal and transverse planes were mostly within $10^{\circ}$, as were found during stair ascending. The hip joint was $2.2^{\circ}$ abducted around initial contact and became

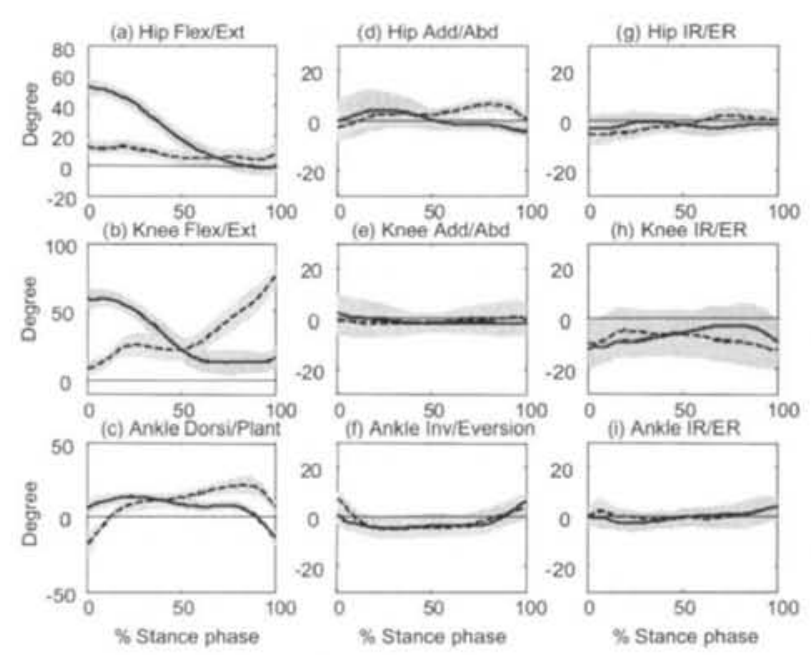

Fig. 2. Ensemble-averaged joint angles in the sagittal (a-c), frontal (d-f) and transverse planes (gh) during stance phase of stair ascent (solid lines) and descent (dashed lines). Shadow areas are one standard deviation from the mean curves. 
adducted after $10 \%$ cycle, reaching a maximum of $6.6^{\circ}$ adduction at $80 \%$ cycle. During the last $20 \%$ cycle, the hip started to abduct and returned to neutral position at toe-off (Fig. 2d). The ankle joint inverted about $7.1^{\circ}$ at initial contact and then everted rapidly to $4.5^{\circ}$ during loading response. It remained at this position until $75 \%$ cycle, when the ankle began inversion again and reached $3.7^{\circ}$ of inversion at toe-off (Fig. $2 \mathrm{f}$ ). The values of the peak moments for the three joints during stair descent were similar to those during stair ascent although the moment curves were different in shapes (Fig. 3d-i). However, the hip adductor moment curve was similar to that during stair ascent albeit with bigger peak values (first peak: $12.5 \%$; second peak: $10.7 \%)$. Hip abductor eccentric power with a maximum of $3.1 \%$ (Watt/BW) was needed during the first $75 \%$ cycle while concentric power with a maximum of $2.4 \%$ (Watt/BW) was necessary during the late stance $(80-100 \%)$, Fig. 4 d. Powers at other joints were much smaller compared to those of the hip muscles.

\section{DISCUSSION}

Stair locomotion is a common functional activity of daily living. This study provided a comprehensive description of the three-dimensional motions of the lower limb joints during this activity. The sagittal kinematics of the lower extremity reported in this study agreed well with those in previous $2 \mathrm{D}$ studies [1 $3]$. The knee moments were in agreement with those reported in previous 3D studies on the knee joint [5-6]. However, the sagittal and coronal components of the knee moments were different from those from previous 2D studies, as were reported in Kowalk et al. (1996). The angular powers also agreed with the literature [12]. Although the stair locomotion in the present study was limited to three steps, the results can be taken as general patterns for normal daily stair movements, as the calculated joint variables were highly reproducible no matter which step in the stairs was selected for data collection [13].

\subsection{Stair Ascent}

Flexion of the hip, knee and ankle of the leading limb according to the dimension of the stairs was required for the appropriate placement of the foot on the upper step at initial contact. This is essential for the body stability required during loading response, when the stance limb accepted gradually the body weight with little movement and increased extensor moments at the joints to increase their stiffness and stability. As the body weight was completely transferred to the stance limb, the hip and knee joints
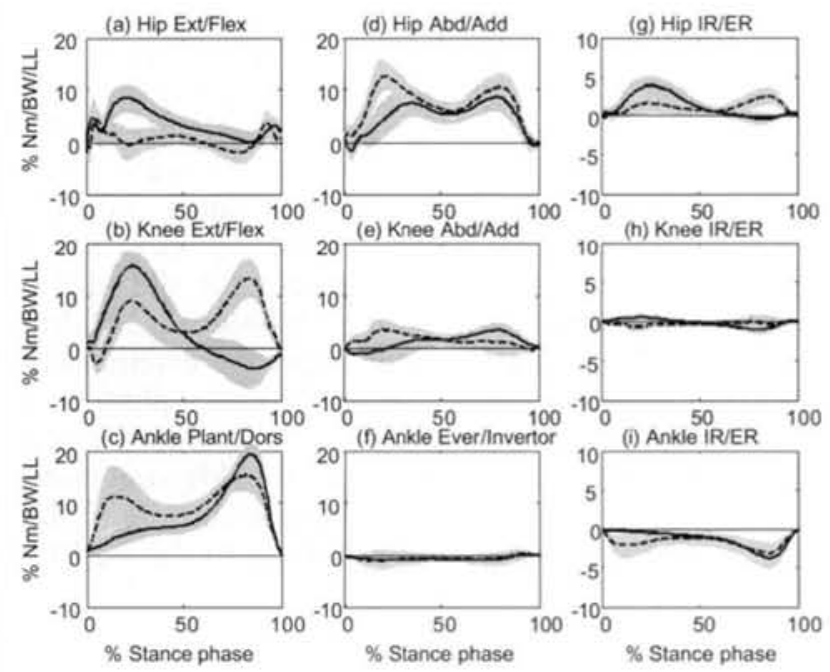

Fig. 3. Ensemble-averaged joint moments in the sagittal (a-c), frontal (d-f) and transverse planes ( $g$ h) during stance phase of stair ascent (solid lines) and descent (dashed lines). Shadow areas are one standard deviation from the mean curves.

of the standing limb gradually extended and exerted extensor concentric muscle power to lift the body up and clear the trailing limb from the ground in order to move to further step. Meanwhile, the leading foot was planted on the step with little movement at the ankle, where only a relatively small plantarflexor moment was necessary to counteract the external dorsiflexion moment due to the advancing body centre of mass (COM). During this period, the activation of the gluteus maximus, quadriceps and gastrocnemius were needed for the extending of the joints but the antagonistic muscles including hamstrings and tibialis anterior were also acting to stabilize the knee joint and to control the anterior rotation of the tibia, i.e. the ankle rocker. The tibilalis anterior remained activated throughout the stance phase to counteract the external plantarflexion moment at the first half of the stance phase and to stabilize the ankle joint while plantarflexors extensively activated for propulsion in the late stance.

Although the ranges of motion of the three joints in the frontal and transverse planes were relatively small, significant joint moments and powers were necessary during stair ascent. The hip abductor moment was the most significant among the others and had two peaks, the first being corresponding to body weight acceptance during loading response and the second to body weight release to the contralateral limb. The ankle was at neutral position at initial contact and then pronated to increase the base of support and to form a looser structure of the foot for the absorption of 
the impact loading during weight acceptance. In late stance, the ankle supinated to form a rigid structure and act as a lever for transmitting propulsive force from the ground to the joints. Joint angular powers in the frontal and transverse planes were less significant compared to the hip abductors. A concentric abductor power of $-0.5 \sim 2.5 \%$ (Watt/BW) at the hip was needed throughout the stance phase except for the initial double stance phase (or loading response). The hip abductor moments were to counteract the external hip adducting moments during the single stance phase and then to transfer the body weight to the contralateral limb at the end of the stance phase. This suggested that the hip abductors played an important role in the body weight transfer from the trailing to the leading limb.

\subsection{Stair Descent}

At initial contact, the three joints of the lower limb were extended to reach the lower step, followed by slight flexions at the knee and ankle to absorb the impact and to accept the body weight. The three joints remained in this position to stabilize the body when the other limb was swinging. When further lowering of the body COM was needed in the second half of the stance phase, the movements in the sagittal plane occurred mainly at the knee and ankle joints in order to have a better control of the upper body, the range of motion of the hip joint being much smaller. The corresponding hip extensor moments were also small, except at initial contact and during propulsion around toe-off, in which accommodation of larger impacts were required. A concentric power at the hip was needed to move the COM forward at initial contact and an eccentric power was used to decelerate the falling of the body COM toward the contralateral limb in late stance.

The extensor moments at the knee and ankle joints had two peaks during stair descent. The first peaks were needed to counteract the externally flexing moment when the COM of the body was behind the joints. The second peaks were required to control smoothly the flexion motion for lowering the body in late stance. According to the muscular powers and EMG profiles, the controlled lowering of the body COM was supported with the eccentric contraction of the extensors in the knee and ankle joints at loading response. Eccentric knee extensor power and eccentric dorsiflexor power in late stance were needed to lift up the foot.

Co-contraction of the rectus femoris and biceps femoris was found in the first half of the stance phase, when controlled knee joint flexion was critical for transferring body weight to the stance limb. Similar results were also found for the tibialis anterior and
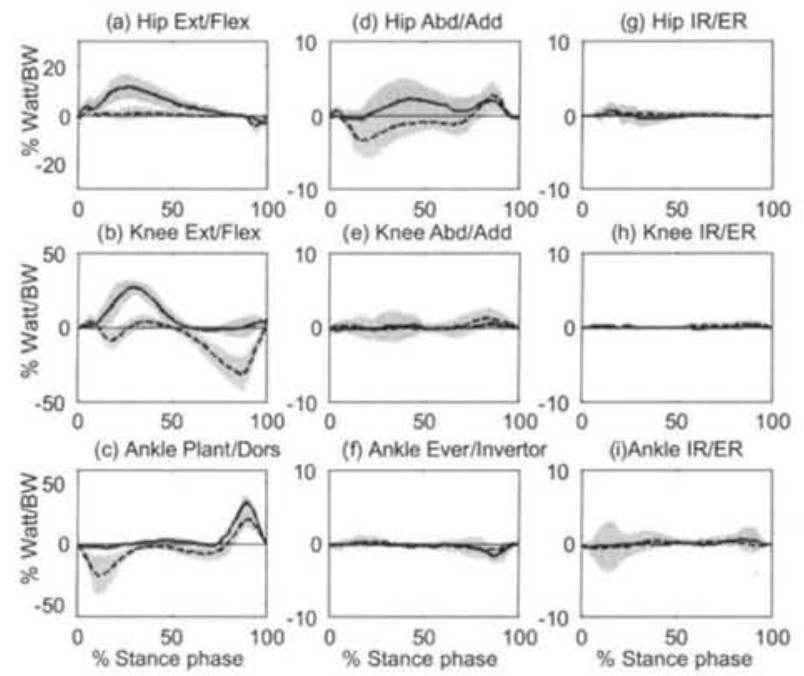

Fig. 4. Ensemble-averaged joint powers in the sagittal (a-c), frontal (d-f) and transverse planes (gh) during stance phase of stair ascent (solid lines) and descent (dashed lines). Shadow areas are one standard deviation from the mean curves.
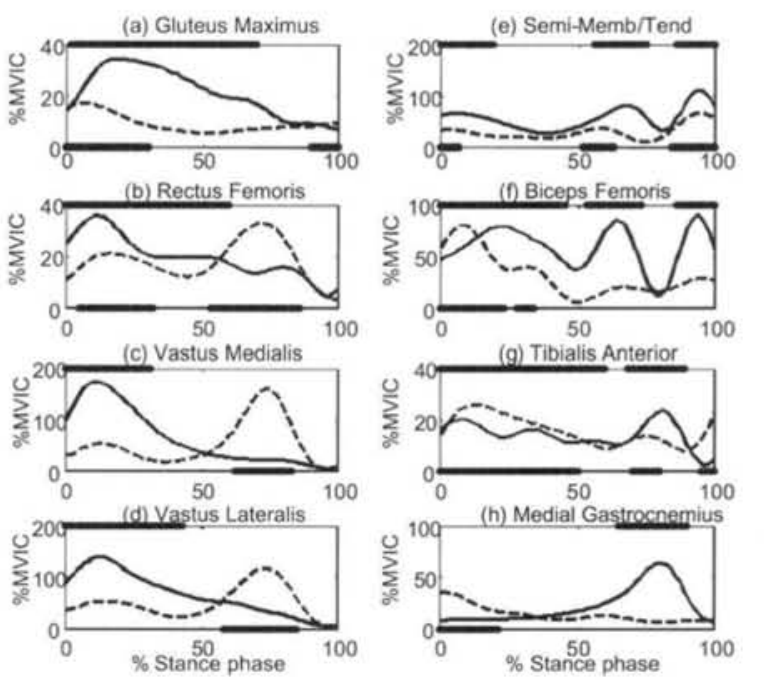

Fig. 5. Ensemble-averaged EMG linear envelops during stance phase of stair ascent (solid lines) and descent (dashed lines). The phasic activity of the muscles are shown as horizontal bars at the top for stair ascent and bottom for stair descent.

gastrocnemius, which were co-activated to carry out a controlled dorsiflexion during the first $20 \%$ cycle. Activation level for gastrocnemius decreased while tibilis anterior remained active to clear the foot during the late stance phase. 
Joint moments and powers in the frontal and transverse planes were much smaller than those in the sagittal plane, except for the hip abductor/adductor. Hip abductor moment demonstrated a two-peak pattern, the first peak being corresponding to the acceptance of body weight transferred from the contralateral side, and the second peak to the control of the body weight transferred back to the contralateral side. An eccentric abductor power at the hip was necessary for the first $75 \%$ cycle to lower slowly the body. A concentric power to shift the body COM to the contralateral limb was required in the late stance, corresponding to the loading response of the cotralateral limb. To have a more stable support of the leading limb at the initial contact on the lower step, not only the three joints of the lower extremity kept in more or less extended position, the ankle and foot also remained supinated to form a rigid lever prepared for the subsequent weight acceptance. After that, the ankle and foot pronated to form a softer structure to accept and absorb the increasing body weight. At the end of the stance, ankle joint supinated again and played a role on transmitting the propulsive force from the ground.

\subsection{Stair Ascent and Descent}

The movement patterns and strategies for stair ascent and descent were quite different. The major differences existed during the initial and terminal double stance phases, i.e. weight acceptance during $0-25 \%$ cycle and weight release during $75-100 \%$ cycle. During these phases, body COM should remain above the supporting trailing limb before it was transferred to the leading limb. During stair ascent, the leading limb accepted the body weight from the trailing limb before it started to extend to lift the body upward to be above the upper step. This resulted in the forward and upward movement of the body COM during this period [4], leaving enough clearance for the trailing foot to swing to the upper stairs. The rising of the body COM was achieved mainly by the concentric contraction of the extensors of the hip and knee joints. During descending, while the leading limb was accepting the body weight from the trailing limb, the knee and ankle joints were flexing to have the body move forward and downward to be above the lower step. This resulted in the forward and downward movement of the body COM during this period [4]. Eccentric joint powers from the hip abductors, knee extensors and ankle plantarflexors were responsible for the control of this movement. Relative big flexion angles at the hip and knee were needed in stair ascent while extended hip and knee were adopted in stair descent, due to obvious requirements from stair dimensions on the formation of the close kinematic chain of the pelvis-leg apparatus.
During weight release (terminal double stance phase), the movement of the COM in stair ascent was mainly controlled by the contralateral limb, which has now become the leading limb, assisted by the plantarflexion of the weight-releasing foot through the concentric contraction of the gastrocnemius. The lowering of the body COM during stair descent was controlled mainly by the knee joint flexion and the eccentric joint powers from the hip abductors, knee extensors and ankle dorsiflexors. Both the hip and knee were required to extend in stair ascent while a relatively high flexed knee and an extended hip were needed in stair descent.

It was generally presumed that the loads in the lower limb are larger during stair descent than stair ascent. From the results of this study, we did not find significant differences in the peak joint moments and powers, except for the peak hip abductor moments. However, longer loading period and eccentric powers were needed in the lower limb during stair descent, which may lead to higher risks of muscle fatigue or strains.

\section{CONCLUSION}

The three-dimensional movements of the lower limb joints during stair ascent and descent were presented. The results of the present study showed that the biomechanics of the lower limb during stair ascent and descent were significantly different. This was due to different safety requirements and kinematic and kinetic constraints from the stairs. The present study provided for the first time a complete description of the biomechanics of the lower extremity while performing stair locomotion. It will be helpful for the planning and evaluation of treatment programs for patients with lower limb problems.

\section{ACKNOWLEDGEMENTS}

The authors gratefully acknowledge the financial supports from the National Health Research Institute (NHRI-EX91-9126EP) and China Medical University (CMC87-PT-03).

\section{REFERENCES}

1. Andriacchi TP, Andersson GBJ, Fermier RW, et al.: A study of lower-limb mechanics during stairclimbing. J Bone \& Joint Surg 1980; 62A: 749-759. 
2. Livingston LA, Stevenson JM and Olney SJ: Stairclimbing kinematics on stairs of differing dimensions. Arch Phys Med Rehabil 1991; 72: 398402.

3. McFadyen BJ and Winter DA: An integrated biomechanical analysis of normal stair ascent and descent. J Biomechanics 1988; 21: 733-744.

4. Zachazewski JE, Riley PO and Krbs DE: Biomechanical analysis of body mass transfer during stair ascent and descent of healthy subjects. J Rehabi Res \& Develop 1993; 30: 412-422.

5. Kowalk DL, Duncan JA and Vaughan CL: Abduction-adduction moments at the knee during stair ascent and descent. J Biomechanics 1996; 29 : 383-388.

6. Yu B, Stuart MJ, Kienbacher T, et al.: Valgus-varus motion of the knee in normal level walking and stair climbing. Clin Biomech 1997; 12: 286-293.

7. Costigan PA, Deluzio KJ and Wyss UP: Knee and hip kinetics during normal stair climbing. Gait \& Posture 2002; 16: 31-37.
8. Kaufmana KR, Hughesa C, Morreya BF, et al.: Gait characteristics of patients with knee osteoarthritis $\mathrm{J}$ Biomechanics 2001; 34: 907-915.

9. Grood ES and Suntay WJ: A joint coordinate system for the clinical description of three-dimensional motions: application to the knee. Trans ASME J Biomech Eng 1983; 105: 136-144.

10. Standards and Terminology Committee of the International Society of Biomechanics. http://www.isbweb.org/standards/index.shtml

11. Winter DA: Biomechanics and motor Control of Human Movement. John Wiley \& Sons, Inc: New York. 2nd ed., 1990; 51-66.

12. Duncan JA, Kawalk DL and Vaughan, CL: Six degree of freedom joint power in stair climbing. Gait \& Posture 1997; 5: 204-210.

13. Yu B, Kienbacher T, Growney ES, et al.: Reproducibility of the kinematics and kinetics of the lower extremity during normal stair-climbing. J Ortho Res 1997; 15: 348-352. 